\title{
Diagnostic Accuracy of MR Mammography in Comparison with Digital Mammography and Sonomammography
}

\author{
Sreenidhi Sedguli ${ }^{*}$ (D), Raghu Srinivasa Gowda ${ }^{1}$, Rupa Ranganathan ${ }^{2}$, Senthil Kumar B ${ }^{3}$
}

\begin{abstract}
Background. Even though the sensitivity of contrast-enhanced breast magnetic resonance mammography (CE-MRM) is consistently high in the range of $94-100 \%$, conventionally, digital mammography and sonomammography continue as standard imaging modalities for the detection and evaluation of breast disease. The objective of the study was to detect additional lesions that go undetected by routine digital mammography and sonomammography using CE-MRM.

Materials and Methods. In a prospective study, 68 patients who came for screening diagnostic mammogram and had breast lesions of Breast Imaging-Reporting and Data System category 3-6 were evaluated. All patients underwent bilateral digital mammography and targeted high-frequency sonomammography of the primary lesion. Those patients who were thought to possibly have breast cancer and to be candidates for surgical management were offered bilateral CE-MRM.

Results. In this prospective study, we included 68 patients (mean age - 50.6 years, range - 30-73 years). A total of 74 lesions were evaluated. In detecting these lesions, digital mammography had a sensitivity of $40.0 \%$, specificity of $100 \%$ and diagnostic accuracy of $63.5 \%$. CE-MRM sensitivity was found to be $71.7 \%$, specificity - $96.6 \%$ and diagnostic accuracy - 83.7\%. Among the 27 additional lesions detected by CE-MRM, histopathological evaluation confirmed only 19 , indicating the sensitivity of $100 \%$, specificity of $85.4 \%$, positive predictive value of $67.8 \%$, negative predictive value of $100 \%$, diagnostic accuracy of $89.2 \%$. Conclusions. The diagnostic accuracy of CE-MRM was found to be $83.7 \%$, with a specificity of $96.6 \%$. CEMRM detected 19 additional lesions that were undetected by either digital mammography or ultrasonography. CE-MRM is sensitive in detecting additional malignant lesions which are not detected by other imaging modalities.
\end{abstract}

Keywords

Screening; Breast Cancer; Contrast-Enhanced Mr Mammography; Ultrasound; Dense Breast

${ }^{1}$ Department of Radiology, Travancore Medical College, Kollam, Kerala, India

${ }^{2}$ Department of Radiology, Kovai Medical Center and Hospital, Coimbatore, Tamil Nadu, India

${ }^{3}$ Golden scans, Anna Nagar, Chennai, Tamil Nadu, India

*Corresponding author: drsreenidhi2005@gmail.com

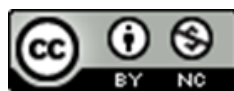

Copyright @Sreenidhi Sedguli, Raghu Srinivasa Gowda, Rupa Ranganathan, Senthil Kumar B, 2022

\section{Introduction}

Breast disease is largely detected by digital mammography (DM) and sonomammography. However, DM misses the detection of breast cancers in up to $30 \%$ cases [1-3]. Denser breast parenchyma, inappropriate imaging technique, radiologist's perceptual errors and subtle malignant features are some of the possible causes of a lesser accuracy of DM $[3,4]$.

Sonomammography (diagnostic or targeted ultrasound) is a high-resolution ultrasound of the breast and it plays an important role in the evaluation of breast cancer [5]. It is effective in differentiating solid and fluid-containing masses. It is useful in the evaluation of clinically suspected breast lesions in women younger than 30 years of age, and as a supplement to conventional mammography in the evaluation of many abnormalities seen on mammograms [2]. It is considered as equivalent to mammography among women with an average risk of breast cancer with a positive family history [6]. Ultrasonography may be useful in detecting occult breast cancer in dense breasts.

Magnetic resonance mammography (MRM) is performed when the diagnosis of cancer is uncertain after mammography and breast ultrasound in patients reluctant to undergo biopsy. An important indication is to exclude synchronous 
cancer in the contralateral breast [3]. Fast 3D gradientecho sequences are used for visualization of changing tissue enhancement after gadolinium injection [7]. Contrastenhanced MRM (CE-MRM) is used as an adjunct to DM and sonomammography [2] and its sensitivity is consistently high in the range of 94-100\% [8].

There were attempts to compare CE-MRM, DM and sonomammography by various researchers [9-11]. As these studies did not consider all three diagnostic modalities in the same setting, in this study, we attempted to compare DM, CE-MRM and histopathological evaluation (HPE).

The objective of the study was to detect additional lesions that go undetected by routine DM and sonomammography using CE-MRM.

\section{Materials and Methods}

In a prospective study, patients presenting to the General Surgery, Oncology and Gynaecology outpatient departments with clinical symptoms of breast cancer or for screening for breast neoplasia were evaluated through DM and sonomammography. At the time of enrolment in the study, patients were counselled that there was a possibility of detecting other lesions during CE-MRM that could lead to additional imaging studies.

\section{Study Centre}

This study was carried at the Department of Radiology of a multi-speciality hospital with the Comprehensive Cancer Centre and a separate Dedicated Breast Centre headed by the radiologist having more than 5 years of experience in breast imaging. The study was done from October 2012 to October 2014

\section{Inclusion Criteria}

- Female patients who came for screening/diagnostic mammogram and who had breast lesions of Breast Imaging-Reporting and Data System (BI-RADS) category 3-6.

- Patients who underwent all three modalities (DM, sonomammography and CE-MRM).

\section{Exclusion Criteria}

- Women who were diagnosed to have BI-RADS category 1-2 on DM and sonomammography.

- Women who were already treated with surgery/ chemotherapy/ radiotherapy and those with no HPE.

- Women who were unable to undergo MR imaging because of a pacemaker, an aneurysm clip, or a metallic foreign body in or near the eye.

- Women who were not willing for consent.

\section{Study Setting}

Each patient and each individual lesion were initially assessed by one of two qualified radiologists with more than five years of experience.

All patients initially underwent bilateral DM with spot and magnification views as indicated. Targeted high-frequency sonomammography of the primary lesion was used to further characterize the morphology, size, and extent. Sonomammography was routinely used to evaluate for other suspicious lesions within each breast and additionally detected lesions were recorded. Those patients who were thought to possibly have breast cancer and to be candidates for surgical management were offered bilateral CE-MRM.

If CE-MRM revealed more extensive breast disease other than the index cancer, the patients would return for a second-look examination with sonomammography. More extensive disease included larger size of index cancer, additional foci of cancer in the same or in other breast quadrants and contralateral lesions.

Second-look sonomammography was performed by the same radiologists who interpreted the CE-MRM images. If the lesion was confirmed as suspicious, a new ultrasound-guided needle biopsy was performed. If the patient refused to undergo a core biopsy, surgical removal was strongly suggested. If the lesion was not seen on secondlook sonomammography, the patient was counselled to remove it if the image was suspicious on CE-MRM, or to have 6-month follow-up CE-MRM if the lesion was less concerning in opinion of the attending breast radiologist. After surgery, all radiographic and pathologic results were examined (Fig. 1).

Lesions, mammographically suspicious for cancer or highly suggestive of malignancy, were sampled by fine-

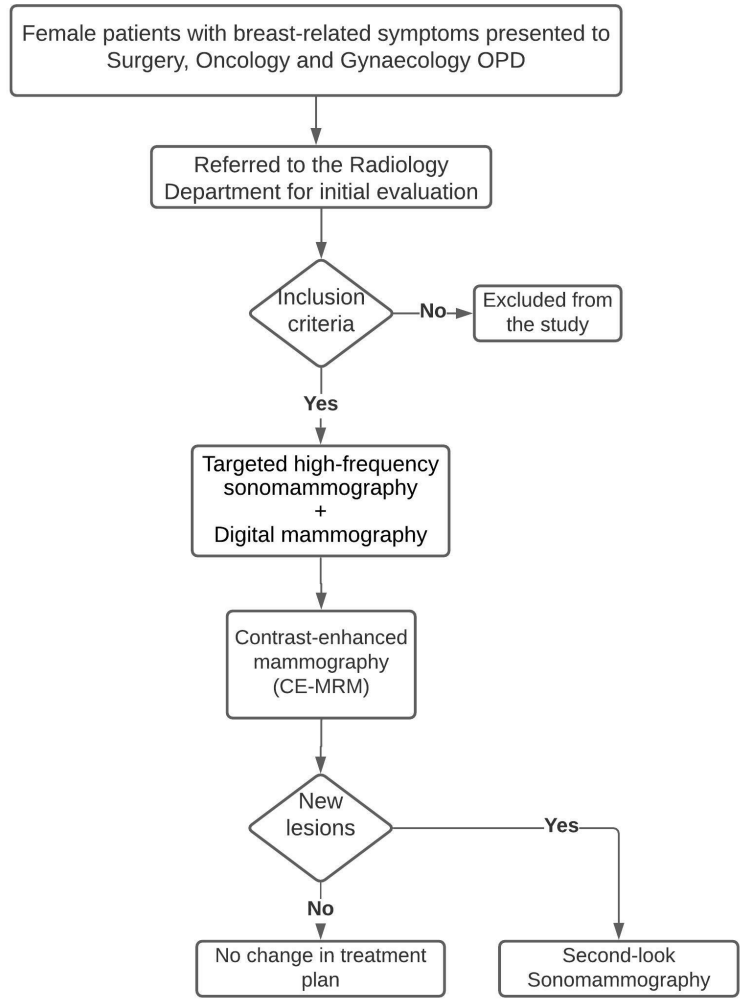

Figure 1. Flowchart of the methodology followed to compare the detection rates of three modalities of investigation - digital mammography, sonomammography, contrast-enhanced breast magnetic resonance mammography. 
needle aspiration cytology (FNAC) or true-cut biopsy if the patient was a candidate for conservation and if the identification of a malignancy at that site would change the surgical approach. Contralateral findings suspicious for or highly suggestive of malignancy were also sampled for biopsy. Biopsy report is considered as a gold standard.

\section{Digital Mammography and Sonomammography}

Bilateral mammography was performed using the Mammomat Inspiration Digital Mammography System. There were included routine craniocaudal and mediolateral oblique views of the breasts and spot compression-magnification views over the area of suspicion. Then, they were examined with the Voluson E8 ultrasound machine - B-mode ultrasound by the same radiologist who was interpreting the mammography images of that patient. Each lesion was characterized and recorded prospectively according to the BI-RADS lexicon as benign (BI-RADS category 1,2 , or 3 ) or suspected malignant (BI-RADS category 4 or 5). Lesion morphology, number of lesions, maximum lesion size dimension and extent were recorded. The breast parenchyma on mammography was also characterized by density as fatty or scattered densities, heterogeneously dense or extremely dense. All the images along with final interpretation report were stored in the picture archiving and communication system (PACS).

\section{CE-MRM Technique}

All positive cases were subjected to CE-MRM imaging within 2 weeks of initial lesion detection. CE-MRM was performed on the SKYRA 3-T MR imaging unit by using a dedicated, four-channel bilateral breast coil, with subjects resting in the prone position.

After the initial pre-contrast sequences, five series of dynamic contrast-enhanced axial T1-weighted 3D fat-suppressed fast-spoiled gradient echo were acquired; (each series 120 sections; each section $-1.3 \mathrm{~mm}$ interpolated to $0.2 \mathrm{~mm}$ intervals; matrix - 416 x 416; 360mm field of view; flip angle $-10^{\circ}$; and 1 acquisition). The initial pre-contrast sequences were acquired before contrast administration. During a subsequent pause of $20 \mathrm{sec}$, a single dose of gadopentetate dimeglumine $(0.1 \mathrm{mmol} / \mathrm{kg}$, Magnevist $)$ was injected at a rate of $1.5 \mathrm{~mL} / \mathrm{sec}$ and was immediately followed by a $20-\mathrm{mL}$ normal saline flush injected at same rate with a power injector. Series 2-5 were then acquired sequentially with no inter-scan delays. Centric spatial encoding was used for all sequences. Reconstruction was done in the coronal and sagittal planes once post contrast images were acquired. Subtraction images generated were used for interpretation. Delayed contrast-enhanced axial with high resolution T1-weighted 3D fat-suppressed fast-spoiled gradient echoes were acquired. Single-voxel spectroscopy of suspicious lesion was done.

The presence or absence of the areas of abnormal enhancement was classified according to the BI-RADS MRI lexicon [12-15]. Bilateral breast imaging was done to include the usefulness of assessing symmetry and screening of the contralateral breast in patients with newly diagnosed breast carcinoma.
In both modalities of evaluation (DM and CE-MRM), the sensitivity, specificity, positive predictive value (PPV), negative predictive value (NPV), and diagnostic accuracy were calculated.

\section{Statistical Analysis}

Discrete variables were analysed for the mean, median, standard deviation (SD), and range; categorical variables were analysed using cross-tabulations - Chi-square test and Fischer's exact analysis. P-value $<0.05$ was considered statistically significant. The calculations were done using SPSS 20 software (IBM Corp., Armonk, NY) from the observed data.

\section{Results}

In this prospective study, 68 patients were involved (Table 1 ; mean age -50.6 years, range $-30-73$ years) and a total of 74 lesions were evaluated. Treatment details are summarized in Fig. 2.

Table 1. Tabulation of patient-related parameters and clinical presentations among patients $(n=68)$ included in the study.

\begin{tabular}{lc}
\hline Parameters & $\mathrm{n}$ \\
\hline Patient age: & \\
30 - 40 years & 13 \\
41 - 50 years & 22 \\
51 - 60 years & 20 \\
Over 61 years & 13 \\
\hline Clinical presentations: & \\
Painless lump & 51 \\
Painful lump & 3 \\
Screening & 3 \\
Painless lump with nipple discharge & 2 \\
Only pain & 2 \\
Only nipple discharge & 2 \\
Skin dimpling & 2 \\
\hline
\end{tabular}

In this study, 47 out of 74 lesions operated on showed ductal carcinoma in situ (DCIS) component in histopathology. Eighteen out of 47 DCIS lesions had pleomorphic/linear branching calcifications and 33 lesions had segmental and linear/ductal non-mass-like enhancement during MRM. Both these patterns were subsequently matched with HPE findings. The sensitivity, specificity and predictive values of DM and MRM in comparison to HPE are represented in Fig. 3. These data suggest that CE-MRM is more sensitive in detecting noncalcified DCIS which are not detected on DM (Table 2). In one patient with significant pleomorphic calcification detected on DM, there was no evidence of non-mass-like enhancement in the corresponding area on CE-MRM. This could be due to extensive calcification.

We also calculated the sensitivity, specificity, PPV, NPV and diagnostic accuracy of DM in combination with CE-MRM which showed the increase in the sensitivity, NPV and diagnostic accuracy as $77.2 \%, 74.3 \%$ and $85.1 \%$, respectively (Table 3 ) as compared to those analysed from individual modalities. 


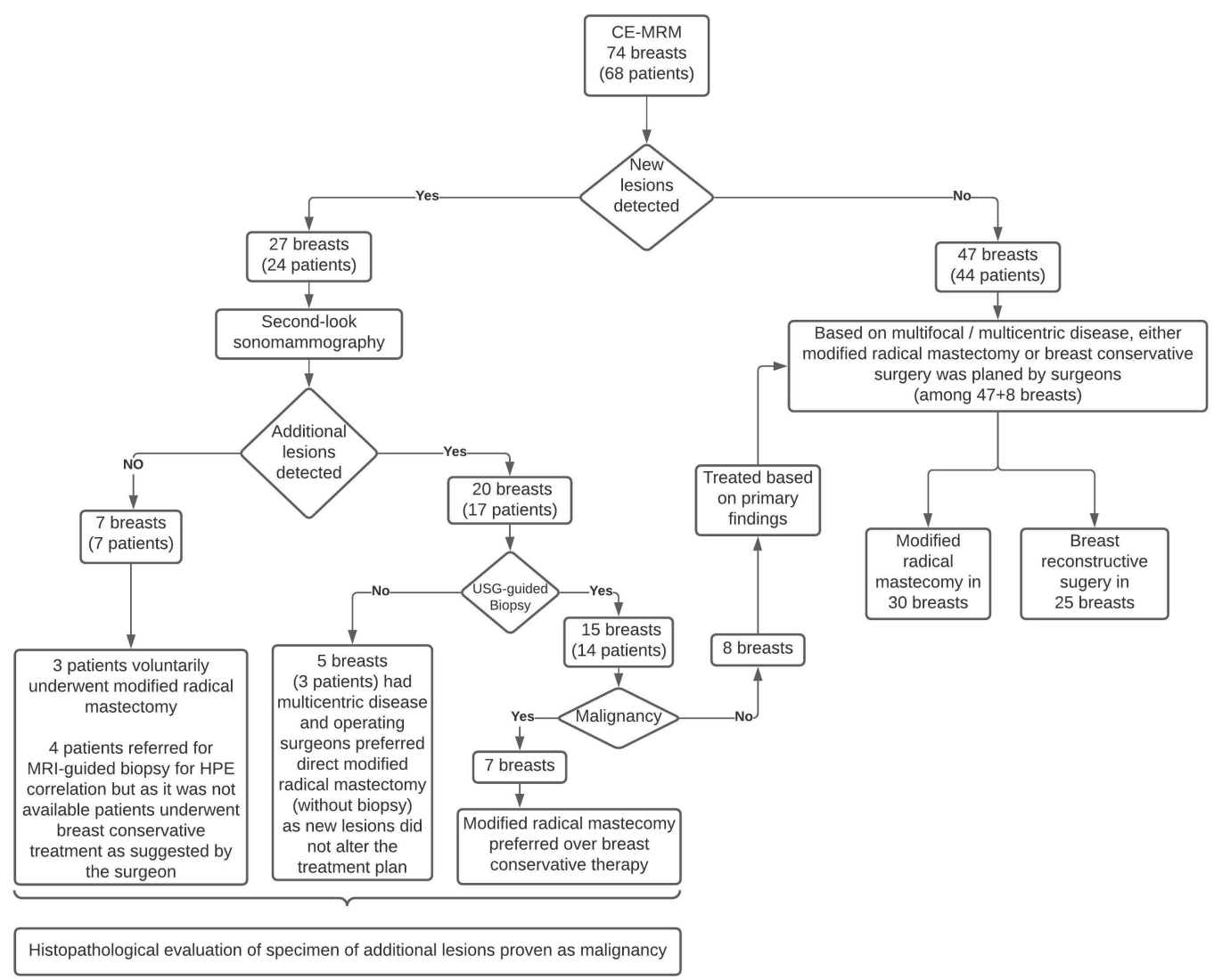

Figure 2. Treatment details of 74 breast lesions (68 patients) included in the study.

Table 2. Cross-tabulation of lesions detected on digital mammography and contrast-enhanced magnetic resonance mammography in comparison to histopathological evaluation $(n=74)$.

\begin{tabular}{|c|c|c|c|c|c|c|c|c|c|}
\hline \multicolumn{5}{|c|}{ Lesions on DM vs Lesions in HPE Cross-Tabulation } & \multicolumn{5}{|c|}{ Lesions on CE-MRM vs Lesions in HPE Cross-Tabulation } \\
\hline & & \multicolumn{2}{|c|}{ Lesions in HPE } & \multirow{2}{*}{ Total } & & & \multicolumn{2}{|c|}{ Lesions in HPE } & \multirow{2}{*}{ Total } \\
\hline & & Detected & Not Detected & & & & Detected & Not detected & \\
\hline Lesions & Detected & 18 & 0 & 18 & Lesions on & Detected & 33 & 1 & 34 \\
\hline on DM & Not Detected & 27 & 29 & 56 & CE-MRM & Not detected & 11 & 29 & 40 \\
\hline Total & & 45 & 29 & 74 & Total & & 44 & 30 & 74 \\
\hline
\end{tabular}

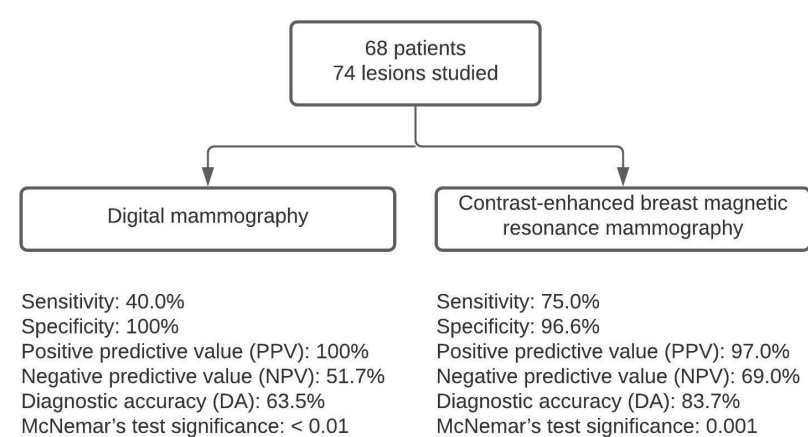

Figure 3. Sensitivity, specificity and predictive values of digital mammography and contrast-enhanced magnetic resonance mammography in comparison to histopathological evaluation.
Table 3. Cross-tabulation of lesions detected by digital mammography in combination with contrast-enhanced breast magnetic resonance mammography in comparison with lesions detected in histopathological evaluation.

\begin{tabular}{|c|c|c|c|c|}
\hline \multirow{4}{*}{$\begin{array}{l}\text { Lesions in DM } \\
\text { and CE-MRM }\end{array}$} & \multirow{4}{*}{$\begin{array}{l}\text { Detected } \\
\text { Not Detected }\end{array}$} & \multicolumn{2}{|c|}{ Lesions in HPE } & \multirow{2}{*}{ Total } \\
\hline & & Detected & Not Detected & \\
\hline & & 34 & 1 & 35 \\
\hline & & 10 & 29 & 39 \\
\hline Total & & 44 & 30 & 74 \\
\hline
\end{tabular}

\section{CE-MRM Detection of Additional Lesions}

Among the 27 additional lesions detected by CE-MRM, HPE confirmed only 19, indicating the sensitivity of $100 \%$, specificity of $85.4 \%$, PPV of $70.4 \%$, NPV of $100 \%$, diagnostic accuracy of $89.2 \%$ (Table 4). MRM had false positive rate of $14.5 \%$ and McNemar's test significance of 0.004 . All these additional lesions were undetected by 
Table 4. Cross-tabulation of additional suspicious lesions detected by contrast-enhanced breast magnetic resonance mammography versus histopathological examination.

\begin{tabular}{l|lccc}
\hline & \multicolumn{3}{c}{ HPE } & \multirow{2}{*}{ Total } \\
\cline { 3 - 5 } CE-MRM addi- & Detected & 19 & 8 & 27 \\
tional lesions & Not detected & 0 & Not detected & \\
\hline Total & 19 & 47 & 47 \\
\hline
\end{tabular}

Table 5. Age less than 50 years and breast density versus additional malignant lesions detected by contrast-enhanced breast magnetic resonance mammography. Cross-tabulation.

\begin{tabular}{|c|c|c|c|c|}
\hline \multirow{2}{*}{$\begin{array}{l}\text { Age }<50 \text { years } \\
\text { with breast } \\
\text { density }\end{array}$} & \multicolumn{2}{|c|}{$\begin{array}{l}\text { Additional lesions } \\
\text { on CE-MRM }\end{array}$} & \multirow{2}{*}{$\begin{array}{l}\text { Odds ratio } \\
(95 \% \mathrm{CI})\end{array}$} & \multirow{2}{*}{ p-value } \\
\hline & Detected & $\begin{array}{c}\text { Not } \\
\text { Detected }\end{array}$ & & \\
\hline $\begin{array}{l}\text { Extremely } \\
\text { dense }\end{array}$ & $\begin{array}{c}4 \\
(30.8 \%)\end{array}$ & $\begin{array}{c}9 \\
(69.2 \%)\end{array}$ & 1.60 & $>0.05$ \\
\hline $\begin{array}{l}\text { Not extremely } \\
\text { dense }\end{array}$ & $\begin{array}{c}5 \\
(21.7 \%)\end{array}$ & $\begin{array}{c}18 \\
(78.3 \%)\end{array}$ & & \\
\hline
\end{tabular}

Note: CI - Confidence Interval.

either DM or ultrasonography, however, picked up by CEMRM. Fig. 4 and Fig. 5 depict two examples of additional lesions detected through CE-MRM.

In addition, it was noted that patients $<50$ years of age had statistically significant extremely dense breast tissue in comparison with patients $>50$ years of age (13 vs. 1 , $\mathrm{p}<0.001)$. Among patients less than 50 years of age with extremely dense breast, CE-MRM detected relatively more additional malignant lesions, however, the inference was statistically not significant (Table 5).

\section{Histopathological Evaluation}

In the study, infiltrating ductal carcinoma and DCIS accounted for majority of cases. Other varieties are tabulated in Table 6.

Table 6. Histopathological evaluation results of the cases included in the study $(\mathrm{n}=74)$.

\begin{tabular}{lc}
\hline Histopathological findings & Abs (n) \\
\hline Infiltrating ductal carcinoma & 37 \\
Infiltrating ductal carcinoma with DCIS & 24 \\
Papillary carcinoma & 3 \\
Invasive papillary carcinoma & 2 \\
Infiltrating ductal carcinoma with invasive papillary car- & 1 \\
cinoma and DCIS & 2 \\
Tubular carcinoma with infiltrating ductal carcinoma & 1 \\
High-grade DCIS only & 1 \\
Lobular carcinoma with lobular carcinoma in situ & 1 \\
Invasive cystic hypersecretory carcinoma & 1 \\
High-grade malignant phyllodes tumour & 1 \\
Borderline phyllodes tumour & \\
\hline
\end{tabular}
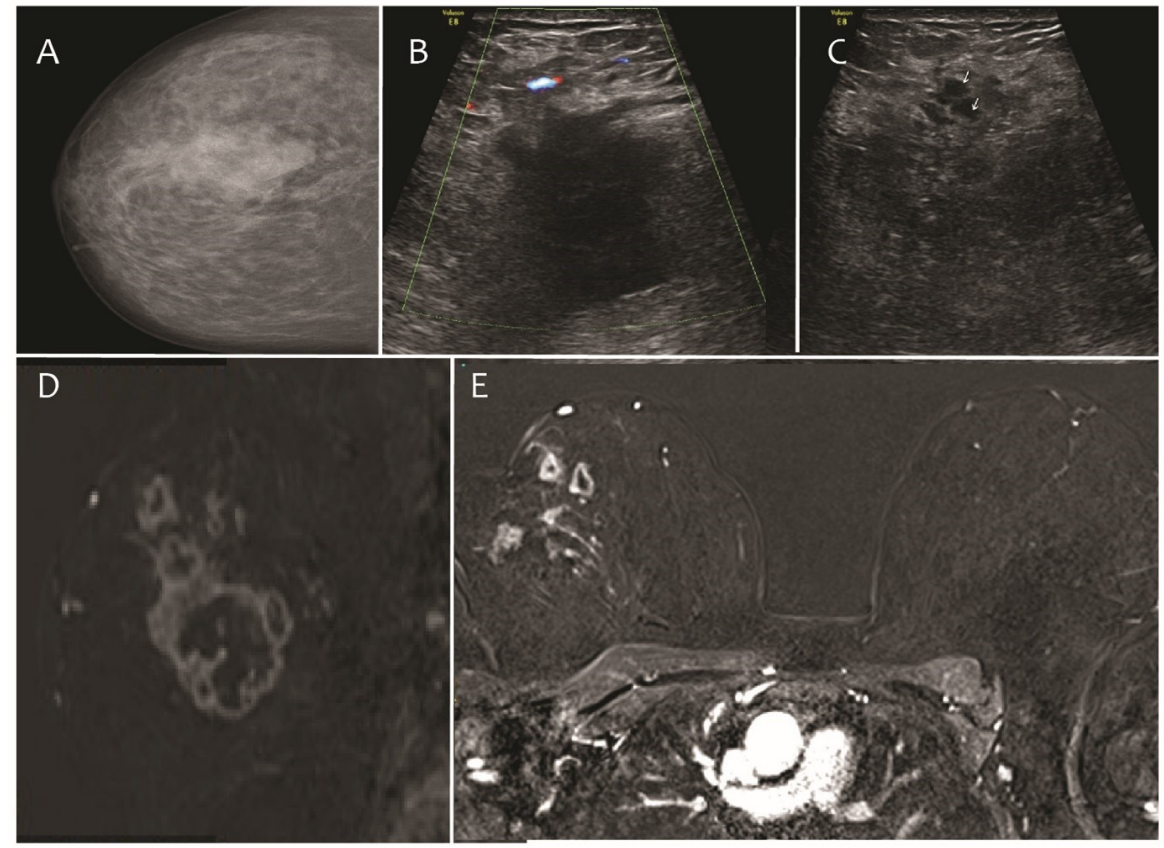

Figure 4. Breast imaging of 49-year-old women with a family history of breast carcinoma presented with complaints of painless lump in the right breast: her digital mammography shows ill-defined hyperdense lesion with architectural distortion (A); sonomammography shows ill-defined hypoechoic lesion with irregular margins and few focal duct ectasias anterior to the index lesion (B and C); whereas contrast-enhanced magnetic resonance mammography clearly shows multiple budding lesions seen predominantly superior and lateral to the index lesion, the farthest additional lesion is more than $4 \mathrm{~cm}$ way from the index lesion suggesting multicentric disease (D and E); histopathology proven as multifocal multicentric infiltrating ductal carcinoma. 

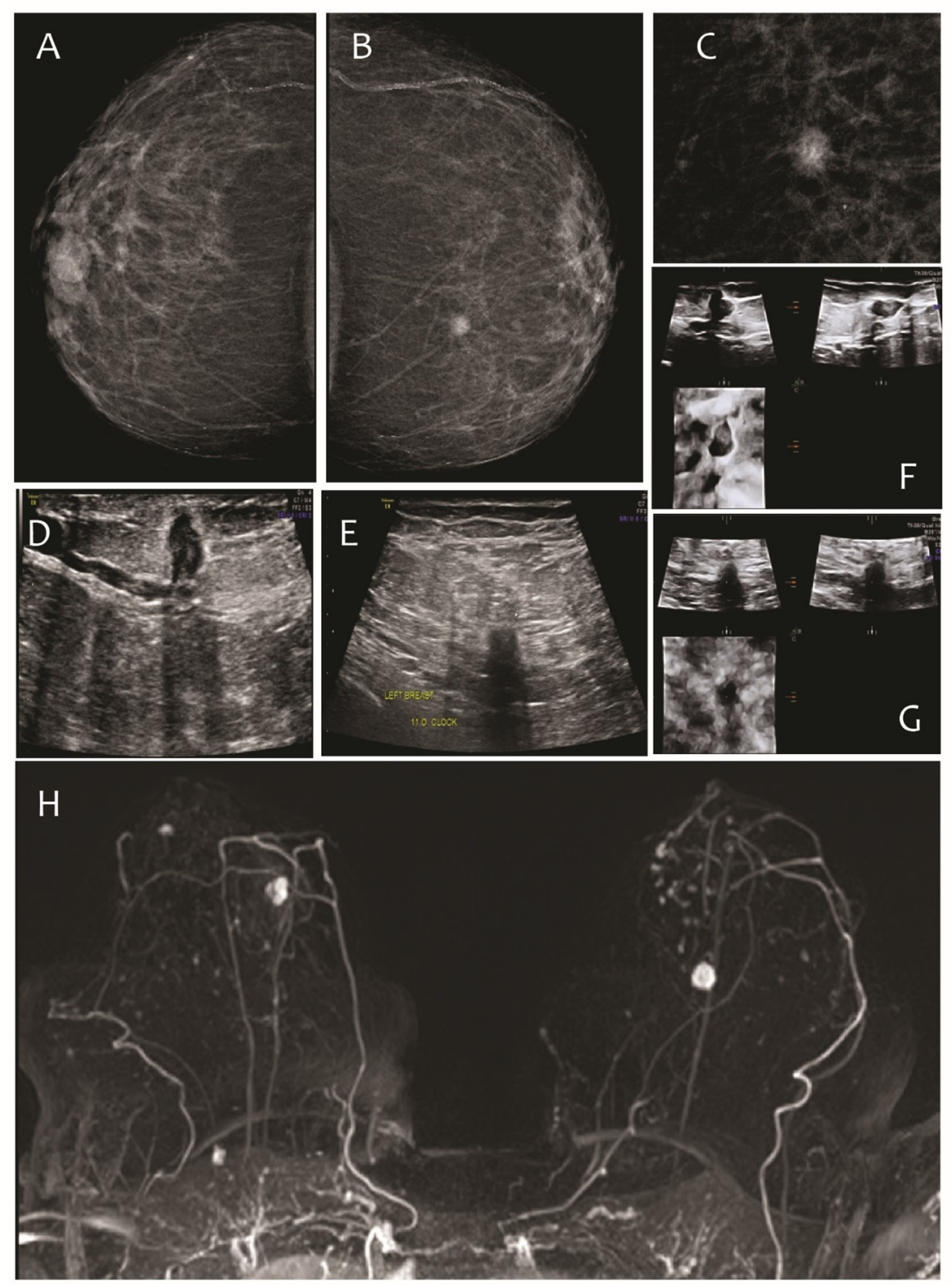

Figure 5. Breast imaging of 69-year-old women with a history of painless lump felt in the right breast for 15 days: digital mammography shows well-defined oval iso-dense lesion in the subareolar region of the right breast (A) and another well-defined macro-lobulated lesion medial to this $(\mathrm{C})$; incident spiculated hyperdense lesion is seen in the left breast (B); on sonomammography, apart from subareolar duct ectasia, vertically oriented intraductal hypoechoic lesion is noted in the right breast ( $\mathrm{D}$ and $\mathrm{F}$ ) which corresponds to the palpable lump and in the left breast, hypoechoic lesion with microlobulated margins is noted $(\mathrm{E}$ and $\mathrm{G})$; contrast-enhanced magnetic resonance mammography, in addition to the above mentioned index lesions in both breasts, shows non-mass-like enhancement with internal clumped enhancement (arrow) in the lower inner quadrant of the left breast $(\mathrm{H})$; histopathology proven as intraductal carcinoma of cribriform type.

\section{Discussion}

Even though sonomammography and CE-MRM have shown more sensitivity than DM for invasive cancer, Berg WA et al., advocated a combination of clinical examination, sonomammography and CE-MRM for more accurate diagnosis of breast lesions [9]. The finding of additional lesions in breast cancer influencing the treatment algorithms should be considered as major additional information during management of breast cancer. Similarly, in the present study, we found 27 additional lesions that were not picked up by mammography and 18 of them were confirmed by histopathology. Fatima $\mathrm{S}$ et al., after evaluating 118 patients with histopathology as gold standard reported a diagnostic accuracy of $92.3 \%$ for breast MR [10]. They advocate the use of CE-MRM as the primary tool for characterization of breast lumps. In the present study, CE-MRM had a diagnostic accuracy of $89.2 \%$. In a similar study on evaluating mammography and CE-MRM, Boné B et al., after evaluating 220 breast cancer patients have inferred 
that these two modalities complement each other [16].

Zhang $\mathrm{Y}$ et al., in a meta-analysis evaluating the diagnostic accuracy of CE-MRM among 11 articles, have concluded that breast MR remains a satisfactory method and should be considered first in all patients with breast lesions [17]. However, the authors failed to provide the overall diagnostic predictive numbers for CE-MRM.

After evaluating 52 women with 120 potential breast lesions, Lee-Felker SA et al. have concluded that contrastenhanced spectral mammography (CESM) is potentially as sensitive as CE-MRM in evaluating newly diagnosed breast cancer [3]. Contrasting these findings, Suter MB et al., have reported a suboptimal sensitivity and specificity for CESM after critically evaluating 8 studies [18]. Therefore, this modality of evaluation was not included in the present study.

High-resolution MRI is used in the evaluation of lesion shape and architecture. Smooth and sharply circumscribed lesions are more likely to be benign [19]. Spiculated and irregular lesions are malignant. If there is a rim enhancement or prominent internal septations, malignancy should be strongly considered. Clumped and linear ductal enhancements are suspicious for malignancy. Associated features like nipple retraction, skin thickening, and lymphadenopathy favour malignancy [19]. As CE-MRM has been associated with overdiagnosis or underdiagnosis, this modality is not universally advocated for screening [20, 21]. Even though many recommendations are in place for screening, breast cancer continues to be major concern and cause of death [22]. For population-based screening with fast outpatient clearance, an abbreviated CE-MRM has been proposed [22]. We performed pre-contrast and dynamic $3 \mathrm{D}$ gradient echo sequences for better lesion characterization in all patients. In a study, Yoo JL et al., found that nonenhanced T2-weighted MR images and kinetic curve assessment could greatly help differentiate malignant breast lesions from benign well-circumscribed breast lesions [23]. The combination of rapid 3D gradient-echo imaging and dedicated breast coil, coupled with a bolus injection of gadolinium dimeglumine had an extremely high sensitivity for breast malignancy as early contrast enhancement was seen in most breast cancers.

In the present study, CE-MRM showed no statistically significant improvement in detecting additional lesions among denser breast women. For screening women with dense breast, sonomammography and tomosynthesis are advocated [24]. However, the selection of one of these two modalities over the others needs clarity [20]. Bakker MF et al., in a two-year prospective study of over 30 thousand women screened for breast cancer, have concluded that the use of supplemental CE-MRM, especially in women with extremely dense breasts, results in fewer interval cancers as compared to mammography alone [25].

Even though CE-MRM has similar diagnostic accuracy, sensitivity and specificity as mammography, it cannot be substituted to routine mammography in a community setting [16]. MR imaging is technically and financially more demanding.

\section{Conclusions}

The diagnostic accuracy of CE-MRM was found to be $83.7 \%$, with a specificity of $96.6 \%$. CE-MRM detected 19 additional lesions that were undetected by either DM or ultrasonography. CE-MRM is sensitive in detecting additional ipsilateral and contralateral malignant tumours which are not detected by other imaging modalities.

\section{Ethical Statement \& Informed Consent}

The Institutional Ethics Committee approved the study at the beginning of the study period. Written informed consent for diagnostic procedures was obtained from each patient.

\section{Conflict of Interest}

The authors declare that no conflicts exist.

\section{Financial Disclosure}

The authors declared no financial support.

\section{References}

[1] Sprague BL, Arao RF, Miglioretti DL, Henderson LM, Buist DSM, Onega T, et al. National performance benchmarks for modern diagnostic digital mammography: update from the Breast Cancer Surveillance Consortium. Radiology. 2017;283(1):59-69. Available from: https://doi.org/10.1148/radiol.2017161519

[2] Iranmakani S, Mortezazadeh T, Sajadian F, Ghaziani MF, Ghafari A, Khezerloo D, et al. A review of various modalities in breast imaging: technical aspects and clinical outcomes. Egyptian Journal of Radiology and Nuclear Medicine. 2020;51(1). Available from: https://doi.org/10.1186/s43055-02000175-5

[3] Lee-Felker SA, Tekchandani L, Thomas M, Gupta E, Andrews-Tang D, Roth A, et al. Newly diagnosed breast cancer: comparison of contrastenhanced spectral mammography and breast MR imaging in the evaluation of extent of disease. Radiology. 2017;285(2):389-400. Available from: https://doi.org/10.1148/radiol.2017161592

[4] Lynge E, Vejborg I, Andersen Z, von Euler-Chelpin M, Napolitano G. Mammographic density and screening sensitivity, breast cancer incidence and associated risk factors in Danish breast cancer screening. Journal of Clinical Medicine. 2019;8(11):2021. Available from: https://doi.org/10.3390/jcm8112021

[5] Yamakanamardi S, Hiremath BV. Accuracy of mammography and sonomammography and its correlation with histopathology in the detection of breast cancer. International Surgery Journal. 2021;8(2):624. Available from: https://doi.org/10.18203/23492902.isj20210374

[6] Harvey JA, Mahoney MC, Newell MS, Bailey L, Barke LD, D'Orsi C, et al. ACR appropriateness criteria palpable breast masses. Journal of the American 
College of Radiology. 2016;13(11):e31-e42. Available from: https://doi.org/10.1016/j.jacr.2016.09.022

[7] Crombé A, Saranathan M, Ruet A, Durieux M, de Roquefeuil E, Ouallet JC, et al. MS lesions are better detected with 3D T1 gradient-echo than with 2D T1 spin-echo Gadolinium-enhanced imaging at 3T. American Journal of Neuroradiology. 2014;36(3):501-507. Available from: https://doi.org/10.3174/ajnr.A4152

[8] Hollingsworth AB. Redefining the sensitivity of screening mammography: a review. The American Journal of Surgery. 2019;218(2):411-418. Available from: https://doi.org/10.1016/j.amjsurg.2019.01.039

[9] Berg WA, Gutierrez L, NessAiver MS, Carter WB, Bhargavan M, Lewis RS, et al. Diagnostic accuracy of mammography, clinical examination, US, and MR imaging in preoperative assessment of breast cancer. Radiology. 2004;233(3):830-849. Available from: https://doi.org/10.1148/radiol.2333031484

[10] Fatima S, Waheed S, Khan MI. Diagnostic accuracy of MR mammography in diagnosing malignant breast lesions taking histopathology as gold standard. Journal of the College of Physicians and Surgeons Pakistan. 2019;29(1):16-18. Available from: https://doi.org/10.29271/jcpsp.2019.01.16

[11] Pereira R de O, Luz LA da, Chagas DC, Amorim JR, Nery-Júnior E de J, Alves ACBR, et al. Evaluation of the accuracy of mammography, ultrasound and magnetic resonance imaging in suspect breast lesions. Clinics. 2020;75:e1805. Available from: https://doi.org/10.6061/clinics/2020/e1805

[12] Aydin H. The MRI characteristics of non-mass enhancement lesions of the breast: associations with malignancy. The British Journal of Radiology. 2019;92(1096):20180464. Available from: https://doi.org/10.1259/bjr.20180464

[13] Spak DA, Plaxco JS, Santiago L, Dryden MJ, Dogan BE. BI-RADS $®$ fifth edition: a summary of changes. Diagnostic and Interventional Imaging. 2017;98(3):179-190. Available from: https://doi.org/10.1016/j.diii.2017.01.001

[14] Magny SJ, Shikhman R, Keppke AL. Breast imaging reporting and data system. In: StatPearls. Treasure Island (FL): StatPearls Publishing; 2021. Available from: http://www.ncbi.nlm.nih.gov/books/NBK459169/

[15] Rao AA, Feneis J, Lalonde C, Ojeda-Fournier H. A pictorial review of changes in the BI-RADS fifth edition. RadioGraphics. 2016;36(3):623-639. Available from: https://doi.org/10.1148/rg.2016150178

[16] Boné B, Péntek Z, Perbeck L, Veress B. Diagnostic accuracy of mammography and contrast-enhanced MR Imaging in 238 histologically verified breast lesions. Acta Radiologica. 1997;38(4):489-496. Available from: https://doi.org/10.1080/02841859709174374
[17] Ren H, Zhang Y. Meta-analysis of diagnostic accuracy of magnetic resonance imaging and mammography for breast cancer. Journal of Cancer Research and Therapeutics. 2017;13(5):862. Available from: https://doi.org/10.4103/jcrt.JCRT_678_17

[18] Suter MB, Pesapane F, Agazzi GM, Gagliardi T, Nigro O, Bozzini A, et al. Diagnostic accuracy of contrast-enhanced spectral mammography for breast lesions: a systematic review and metaanalysis. The Breast. 2020;53:8-17. Available from: https://doi.org/10.1016/j.breast.2020.06.005

[19] Kuhl CK, Strobel K, Bieling H, Leutner C, Schild HH, Schrading S. Supplemental breast MR imaging screening of women with average risk of breast cancer. Radiology. 2017;283(2):361-370. Available from: https://doi.org/10.1148/radiol.2016161444

[20] Berg WA. Current status of supplemental screening in dense breasts. Journal of Clinical Oncology. 2016;34(16):1840-1843. Available from: https://doi.org/10.1200/JCO.2015.65.8674

[21] Welch HG, Prorok PC, O’Malley AJ, Kramer BS. Breast-cancer tumor size, overdiagnosis, and mammography screening effectiveness. New England Journal of Medicine. 2016;375(15):1438-1447. Available from: https://doi.org/10.1056/NEJMoa1600249

[22] Kuhl CK. Abbreviated breast MRI for screening women with dense breast: the EA1141 trial. The British Journal of Radiology. 2018;91(1090):20170441. Available from: https://doi.org/10.1259/bjr.20170441

[23] Yoo JL, Woo OH, Kim YK, Cho KR, Yong HS, Seo BK, et al. Can MR imaging contribute in characterizing well-circumscribed breast carcinomas? RadioGraphics. 2010;30(6):1689-1704. Available from: https://doi.org/10.1148/rg.306105511

[24] Tagliafico AS, Calabrese M, Mariscotti G, Durando $\mathrm{M}$, Tosto $\mathrm{S}$, Monetti $\mathrm{F}$, et al. Adjunct screening with tomosynthesis or ultrasound in women with mammography-negative dense breasts: interim report of a prospective comparative trial. Journal of Clinical Oncology. 2016;34(16):1882-1888. Available from: https://doi.org/10.1200/JCO.2015.63.4147

[25] Bakker MF, de Lange SV, Pijnappel RM, Mann RM, Peeters PHM, Monninkhof EM, et al. Supplemental MRI screening for women with extremely dense breast tissue. New England Journal of Medicine. 2019;381(22):2091-2102. Available from: https://doi.org/10.1056/NEJMoa1903986

Received: 2021-09-15

Revision Requested: 2021-11-04

Revision Received: 2021-12-22

Accepted: 2022-01-18 